\title{
, Effect Of Temperature On The Dynamic
}

Response Of Adhesively Mounted

\section{Accelerometers}

${ }_{4}$ Andrea Spaggiari ${ }^{1}$ and Marco Cocconcelli ${ }^{2}$

${ }^{1}$ Department of Engineering Sciences and Methods, University of Modena and Reggio

Emilia, Italy, E-mail: andrea.spaggiari@unimore.it

${ }^{2}$ Department of Engineering Sciences and Methods, University of Modena and Reggio

Emilia, Italy, E-mail: marco.cocconcelli@unimore.it

\section{ABSTRACT}

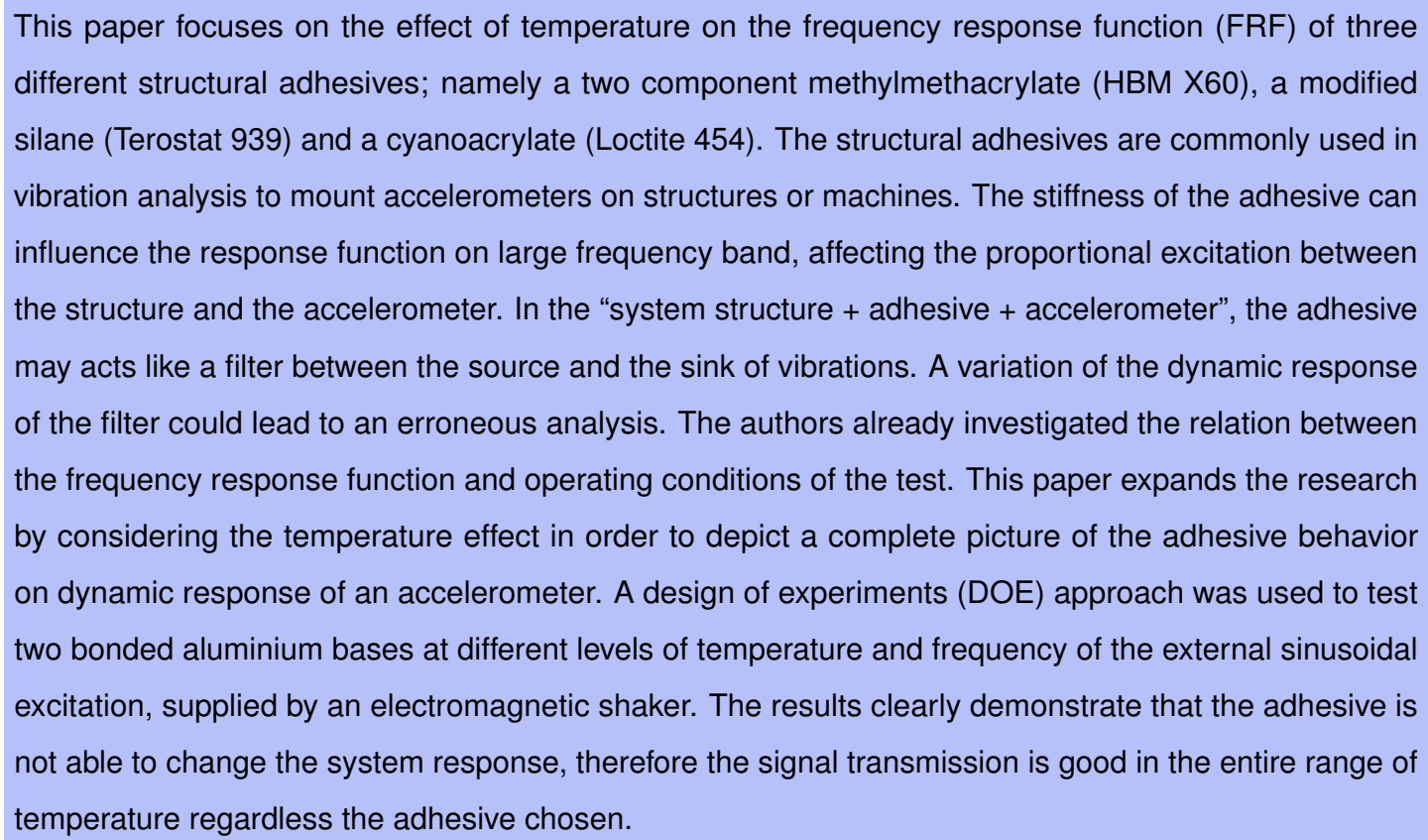

Keywords: Temperature, Mechanical properties of adhesives, Accelerometers mounting, Vibration transmissivity, Experimental testing

\section{INTRODUCTION}

Accelerometers are widely used in reliability and maintenance services to perform analysis and condition monitoring of mechanical components and systems. Most common faults in machines are due to rotating components, such as bearings, gears and shafts. The coupling between the rotating part and the frame of the machine is subject to wear, to fatigue effects, poor lubrication or changing environmental conditions. 
As far as the wear increases the mechanical component breaks, causing unexpected stops of the machine and subsequent economic loss due to lack of production. The accelerometer is a sensor that detects and acquires vibrations of the component it is fixed to, allowing an early detection of the wear before it causes severe damage to the machine, avoiding unexpected stops. Among the so-called Non-Destructive Testing techniques [1], the vibration analysis has a relatively low costs for the sensor and an easy set-up on the machine. All the vibration measurements are normally performed by means of a piezoelectric accelerometer or based on Micro Electro-Mechanical Systems (MEMS) technology. The development of both MEMS and piezoelectric technology leads to better products, resistant to environmental agents, with small dimensions and a great bandwidth. The application of MEMS in many technological products, such as smartphones and gaming application makes these devices extremely low price and very interesting for the vibration monitoring. The introduction of the piezoelectric accelerometer was in the " 50 s while MEMS technology comes lately, thus a relevant amount of papers on signal processing can be found in technical literature, setting the state of the art on vibration analysis so far [2]. Despite the thousands of papers dealing with the problem of vibration signal processing, there are only few papers which focus on a correct setup of the vibration sensors, even though it is crucial for every accelerometer applications [3]. This practical aspect is mainly based on the information retrievable in university courses on vibrations analysis, either on personal experience or to information given by the accelerometer suppliers [4-6]. The supplier' guidelines usually focus on a specific aspects of the accelerometer setup, the mounting between the sensor and the surface of the component. The main solutions available are: screw mounting, stud mounting, magnetic mounting, adhesive mounting and probe mounting. Each method has a specific field of application depending on: the working temperature, the mounting surface conditions, the accessibility to the specific mounting point, etc. A detailed description of all the mounting techniques could be find in classic handbook on shock and vibration [7]. Among other techniques, the adhesive and the screw/stud mounting are the most typical. These mounting techniques result in a rigid connection with high stiffness and wide frequency range response. Compared to screw/stud mounting, structural adhesives are more reliable in the fastening of sensors and accelerometers, since they provide a simple and quick mounting without the need of permanent mechanical processing, such as threaded holes, on the chassis of the machine as stated by Harris [7] as well, while the stud/screw mounting allows a quicker setup on the sensor. In a previous work [8], the authors focuses on the adhesive mounting of accelerometers, assessing experimentally the dynamic response of three different adhesives which cover the most common type of structural adhesive used in on-field applications; namely a two component methylmethacrylate, HBM X60 [9], a cyanoacrylate, Loctite 454, [10] and a modified silane, Terostat 939 [11]. Often it is believed that "soft and rubbery" adhesives, which works above their glass transition temperature, like the modified silane could not be used in this type of application. Secondary objective of the paper is to prove if this sentence, retrievable in literature but with little evidence, is true. By means of an electrodynamic shaker, a design of experiment approach was proposed consisting of three variables in the experimental plan: adhesive type, frequency and amplitude of the vibration signal. The main results proved that the transfer 
function of the adhesive layer does not distort the signal regardless of the type of adhesive. In this paper, a further step of that research is proposed, investigating the influence of the temperature on dynamics response of the above-mentioned adhesives. The effect of temperature on the adhesives was studied in technical literature [12] and also by other authors [13-16], but scarce information is retrievable on their viscoelastic properties at different temperature when in thin films. The global information is related to their polymeric nature, therefore softer adhesive are more affected by temperature and tend to relax more than the stiffer ones when temperature increase. The aim of the paper is to estimate which is the effect of the adhesive film used to join the parts in terms of vibration monitoring and signal transmission. The adhesives are used to join two aluminium bases, the first one connected with a threaded coupling to an accelerometer and the second one coupled with the head of electromagnetic shaker. The specimens are placed in climatic chamber together with the shaker and the sensors. The description of the experimental procedure and the detailed experimental set up are shown in Section 2.3. The Materials and Method section describes as well the design of experiment approach used. It consists of four variables in the experimental plan: adhesive type, frequency, amplitude of the control signal and temperature of the climatic chamber. The amplitude and the frequency are chosen in order to explore the entire range of the electromagnetic shaker available in the laboratory, while the temperature spans the range available on the climatic chamber $\left(23^{\circ} \mathrm{C}-80^{\circ} \mathrm{C}\right)$. A finite element modal analysis, reported in Section 2.4, was carried out in order to estimate numerically the influence of the adhesive layer on the resonant modes of the structures. The results confirm the little influence of the adhesive layer, due to its very low thickness. The Section 3.2 shows the detail of the post processing procedure carried out on the experimental data. The signal is post-processed through a LabVIEW environment and four significant output parameters are extracted: the spectral amplitude of the excitation frequency (Spectral Amplitude - SA), the percentage of signal energy stored at the excitation frequency (Stored Energy - SE), and their weight percent over a reference specimen (Spectral Amplitude Ratio - SAR - and Stored Energy Ratio - SER - respectively). These data are elaborated with a statistical software to evaluate which variable affects the system responses and which is the adhesive's effect on the vibration monitoring. The Section 3.3 exploits the ANOVA (Analysis of Variance) technique to estimate which are the most significant effects on the responses by means of half normal plots and variable interactions diagrams. In the conclusions we present some interesting consideration about the adhesive bonding of the accelerometers and some practical hints useful in laboratory and industrial environment are presented.

\section{METHODS AND MATERIALS}

\subsection{Design of Experimental plan}

The Design of Experiments (DoE) method was developed to optimize the experimental tests especially for multiple variables involved in a problem [17]. The same principles can be also stretched to numerical studies [18], treating each numerical analysis with a different set of levels and variables as a virtual experiment. In the present work the DoE technique was used to assess the effect of the temperature and of 
the input excitation on the dynamic mechanical response of adhesive mounting accelerometers at different working conditions. Four factors were considered in the analysis, namely:

1. Adhesive type, $G$

2. Excitation amplitude, $A$

3. Temperature, $T$

4. Excitation frequency, $f$

A previous paper of the authors [8], analyzed the effect of the frequency and the amplitude on different adhesive bonding, although the chosen levels were different. A cross-check of the results with the ones obtained in the previous work, will be still possible.

The levels of the excitation frequency have been chosen based on a resonance test on a reference specimen at room temperature. The shaker was excited by a linear sine sweep signal in all the shaker frequency range, i.e. from 0 to $12 \mathrm{kHz}$, with a rate of $1 \mathrm{kHz} / \mathrm{s}$. The acquired vibration in time domain is shown in the left part of Fig. 1, revealing the presence of a resonance peak at 7 seconds. The right part of Fig. 1 shows the corresponding frequency spectrum, fixing the resonance at $7485 \mathrm{~Hz}$. It is worth noting that the high amplitude at low frequency is not a resonance of the system, but a limit in the performances of the shaker, optimized to work at mid-high frequencies.
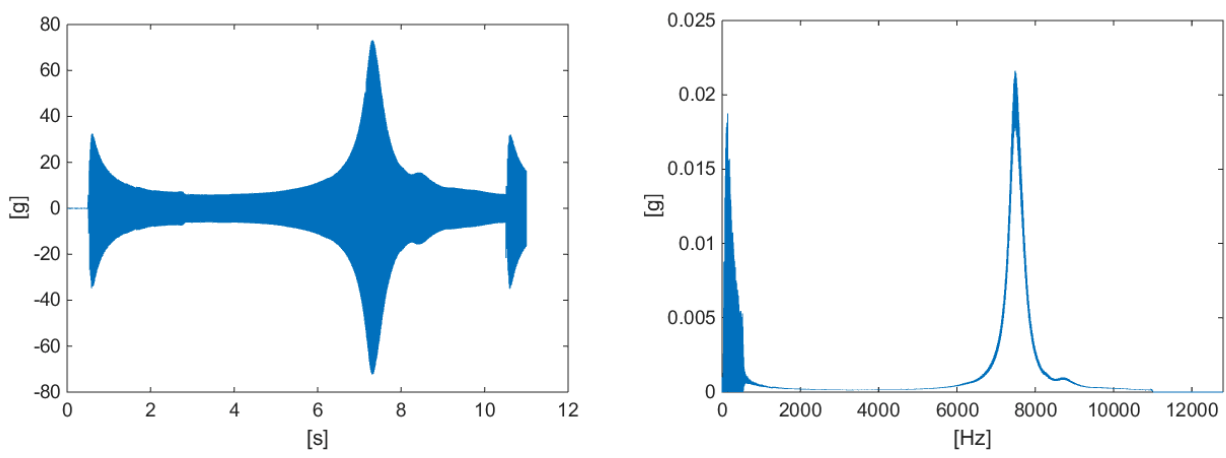

Figure 1. Frequency sweep and resonant peak of the acquisition test rig

Based on the sweep test, the three levels of excitation frequency $f$ are chosen viz. $15 \mathrm{~Hz}, 3500$ $\mathrm{Hz}, 10000 \mathrm{~Hz}$ in order to span the entire range of the accelerometer [19], taking into account also the non-linear behavior at low frequency. It was decided to consider the low frequency range even though the input signal is not clean for two main reasons. The first reason is that at low frequency we have an higher amount of energy and therefore is interesting to monitor these bandwidth. The second reason is that the desired behaviour of the adhesive should not be dependent on the input signal, no matter how bad or distorted is. The values chosen for the excitation amplitude are expressed as a percentage of the maximum amplitude provided by the shaker, i.e. $50 \%$ and $100 \%$. The temperature is the core factor of this analysis, so four levels are chosen to better highlight its influence in the tests: $23^{\circ} \mathrm{C}$ (room temperature), $40{ }^{\circ} \mathrm{C}$, 
$60^{\circ} \mathrm{C}, 80^{\circ} \mathrm{C}$. We decided not to exceed $80^{\circ} \mathrm{C}$ since it is practical limit for many rotating machineries driven by electrical motors. Each specimen was stored in the climatic chamber for a proper time before the test, in order to heating up the aluminum specimen checking the surface temperature by means of a infrared thermometer. The adhesive type are the same four used in [8]: G1 is the commercial superglue (Loctite 454), the G2 is an elastic adhesive modified silane (Terostat 939), both produced by Henkel Adhesive, Cerano (NO), Italy. The G3 is a very stiff two components methylmethacrialte adhesive (HBM X60), produced by HBM, Milan, Italy and G4 is a reference configuration in which there is no adhesive but continuum material. A Dynamic Mechanical Analysis (DMA) on the selected adhesives would have been useful, but in literature we could not trace precise information about the viscoelastic behaviour of the adhesives chosen. Moreover the adhesive behaviour in bulk form and in thin film is pretty different [20-23] so the eventual information given by a DMA in bulk are not immediately applicable to thin adhesive layers. Qualitatively the adhesives have very different viscoelastic behaviour, as retrievable on the basis of the chemistry and on the datasheets of the polymers involved [12,16,24]. The stiffer adhesives, which works below the glass transition temperature which is $137^{\circ} \mathrm{C}$ for $\mathrm{G} 1$ see [10] and about $150^{\circ} \mathrm{C}$ for G3, see [9] have limited viscous effect, while the softer one (G2) is quite viscoelastic, since it works in its rubbery state at room temperature [11]. A summary of the variable levels is reported in Table 1. Each specimen is made by two cylindrical aluminium blocks, connected with a thin layer of adhesive, the lower one is screwed to the shaker, the upper one has a threaded connection for the accelerometer. In the reference configuration (level 4 of "adhesive type" variable in Tab. 1), there is no adhesive between the two blocks but a unique aluminum cylinder with double mass of a single block, in order to keep the same nominal natural frequency. The authors decided to set the adhesive layer thickness at a constant value of $0.05 \mathrm{~mm}$ and then to exclude it from the experimental plan even though it is important in the adhesive stiffness and strength [12-17]. The adhesive thickness is typically an uncontrolled parameter in a practical application of a bonded accelerometer and, the thickness is controlled by the surface roughness only. The description of the deposition of the adhesive layer and the curing process is reported in Section 2.3

\begin{tabular}{lcccc}
\hline Variables & Level 1 & Level 2 & Level 3 & Level 4 \\
\hline Temperature, $T$ & $23^{\circ} \mathrm{C}$ & $40^{\circ} \mathrm{C}$ & $60^{\circ} \mathrm{C}$ & $80^{\circ} \mathrm{C}$ \\
Frequency, $f$ & $15 \mathrm{~Hz}$ & $3500 \mathrm{~Hz}$ & $10000 \mathrm{~Hz}$ & \\
Amplitude, $A$ & $50 \%$ & $100 \%$ & & \\
Adhesive type, $G$ & Loctite 454 & Terostat 939 & HBM X-60 & None (solid) \\
\hline
\end{tabular}

Table 1. Levels of the variables considered in the DOE

\section{(1)}

co

b

exp fred

A full factorial plan is adopted, with two replicates for each experimental plan. This approach is combined with a blocking procedure to take into account the different bonding of the adherends. The blocking procedure is a useful tool, used in the DoE approach, in order to avoid any influence of the experimental set up or the operator, as described in [17, 18, 25]. Four different temperatures, three frequencies, two amplitudes, four adhesives and two replicates lead to a total amount of 144 experiments 
on the bonded configuration, which can be used to estimate the influence of the primary variables and the interactions. Further 48 additional experiments on the reference configuration were carried out, leading to a total of 192 experiments. We exploited the software Design Expert in order to build the set of experimental test to be carried out and to randomize the order of the experiments. The software was also used to post process some of the results using mainly an analysis of variance technique (ANOVA).

\subsection{System response}

The statistical influence of the variables is evaluated in terms of four system responses. The shaker excitation is a sinusoidal wave at a given frequency, therefore the amplitude of the corresponding spectral component is the main output choice. The two main of the outputs of the experiments are:

- Spectral amplitude at excitation frequency (SA)

- Percentage of signal energy stored at excitation frequency (SE)

The SA is obtained by applying the Fast Fourier Transform to the vibratory signal, considering the amplitude of the measured signal at the excitation frequency, the SE is the energy of the vibratory signal at the excitation frequency divided by the total energy of the system.

Other two outputs are computed dividing the values of the experimental points by the value of the reference configuration (on average). The block with double mass and no adhesive is taken as reference configuration. These outputs are not dependent on the system configuration and allow the adhesive effect to be compared more efficiently. The two additional parameters are:

- $S A R=S A / S A_{\text {no_adhesive }}$ Spectral amplitude ratio (SAR)

- $S E R=S E / S E_{\text {no_adhesive }}$ Stored energy ratio (SER)

The four parameters (SA, SE, SAR, SER) were firstly proposed in [8].

\subsection{Experimental set-up}

The experimental set-up consists in the test specimens, a climatic chamber, a small electrodynamic shaker, a monoaxial accelerometer, a data acquisition board and a function generator. Table 2 summarizes the model and the characteristics of the components.

\begin{tabular}{|c|c|c|}
\hline Component & Model & Specifications \\
\hline Climatic chamber & homebuilt & $\begin{array}{l}2 \times 600 \mathrm{~W} \text { fan heater - PT100 temperature sensor, } \\
\text { Ascon hysteresis controller, precision } 23^{\circ} \mathrm{C}\end{array}$ \\
\hline Shaker & ModalShop K2004E01 & $20 \mathrm{~N}$ peak sine force, frequency up to $11 \mathrm{kHz}$ \\
\hline Accelerometer & PCB 353B18 & Monoaxial, freq. range $1-10 \mathrm{kHz}( \pm 5 \%), 10 \mathrm{mV} / \mathrm{g}$ \\
\hline DAQ & NI USB-9162 + NI-9233 & $50 \mathrm{kS} / \mathrm{s}$ per channel, 24-Bit IEPE \\
\hline Function generator & Rigol DG1022 & 2 channels, $20 \mathrm{MHz}$ waveform generator \\
\hline
\end{tabular}

Table 2. Specifications of the components used in the experiment.

All the specimen blocks are built from a single aluminum bar, with a circular cross section of $10 \mathrm{~mm}$ of diameter. Each block is $20 \mathrm{~mm}$ high, except the reference block that has a double height $40 \mathrm{~mm}$, being 
negligible the adhesive weight. The size of the blocks is chosen to ensure space for the threaded hole, but still keeping enough mass in order to have a stiff specimen. All the blocks have been threaded to be coupled with the shaker head and the accelerometer by means of an adaptor. The blocks were bonded using a jig to maintain alignment between the upper and lower one and a small dead weight $(0.1 \mathrm{~kg})$ was used to apply a sufficient pressure to the entire adhesive layer. The adhesive fillet is removed with a cutter immediately after the applications, in order to avoid any extra mass and due to the very fast curing (few seconds) of the Loctite 454 and XBM X60. In order to achieve complete polymerization we kept all the joints at room temperature for $48 \mathrm{~h}$, to ensure a proper curing of all the three adhesives. The estimated nominal thickness is $0.05 \mathrm{~mm}$. The eight specimens ( 4 adhesive levels and 2 replications), the accelerometer and the acquisition board are shown in Fig. 2. The shaker is provided with an embedded amplifier, open-chain controlled with 0-1 VRMS signal supplied by a Rigol function generator. The shaker is equipped with an amplitude gain which can be selected by the user, but in this experiment we decided to keep it constant for all the tests. The accelerometer is acquired by means of a NI-9233 board which is specifically designed for IEPE devices. The shaker is fixed to the ground of the climatic chamber, while the shaker body is connected to its fixed frame by vises. In each test the head of shaker moves harmonically with characteristics listed in Table 2 . The amplitude is not measured in absolute "g" value, but it is expressed by a percentage of the maximum control voltage, i.e. with a Vpeak value of $1 \mathrm{~V}$ for the $100 \%$ of amplitude and $0.5 \mathrm{~V}$ for the $50 \%$ amplitude. The sampling frequency is $50 \mathrm{kHz}$ and the acquisition time is 3 seconds. The acquisition system waits two seconds before starting to avoid acquisition of undesirable transient effects of the shaker. The climatic chamber is home built, made in PVC insulating panels (12mm thick) with two fan heater (600W each) controlled by an Ascon controller. The controller ensures the desired temperature by means of a PT100 thermocouple and implements a threshold control with a mean error of $0.5^{\circ} \mathrm{C}$. The fans, integrated in the heater ensure an uniform temperature distribution in the climatic chamber. Figure 3 shows the final setup of the shaker in the climatic chamber.

\subsection{Finite Element Model}

This section describes a preliminary analysis of the system carried out by means of a finite element modal analysis. The aim of the parametric analyses is to assess the theoretical influence of the adhesive layer on the natural frequencies of the system considered. Four different analyses were carried out: three different adhesives and the reference configuration are modelled. The finite element modal analyses are carried out by using Abaqus 6.141 FEA solver. The model is composed by two aluminum blocks with the exact dimensions of the specimen described in the previous sections, bonded by a $0.05 \mathrm{~mm}$ thick adhesive layer. The mesh continuity guarantees that no contact elements disturb the transition between different materials. The model used for the reference configuration shares the same mesh with the other three bonded models. The accelerometer weight is considered and the threaded connection of the accelerometer is modelled as a 


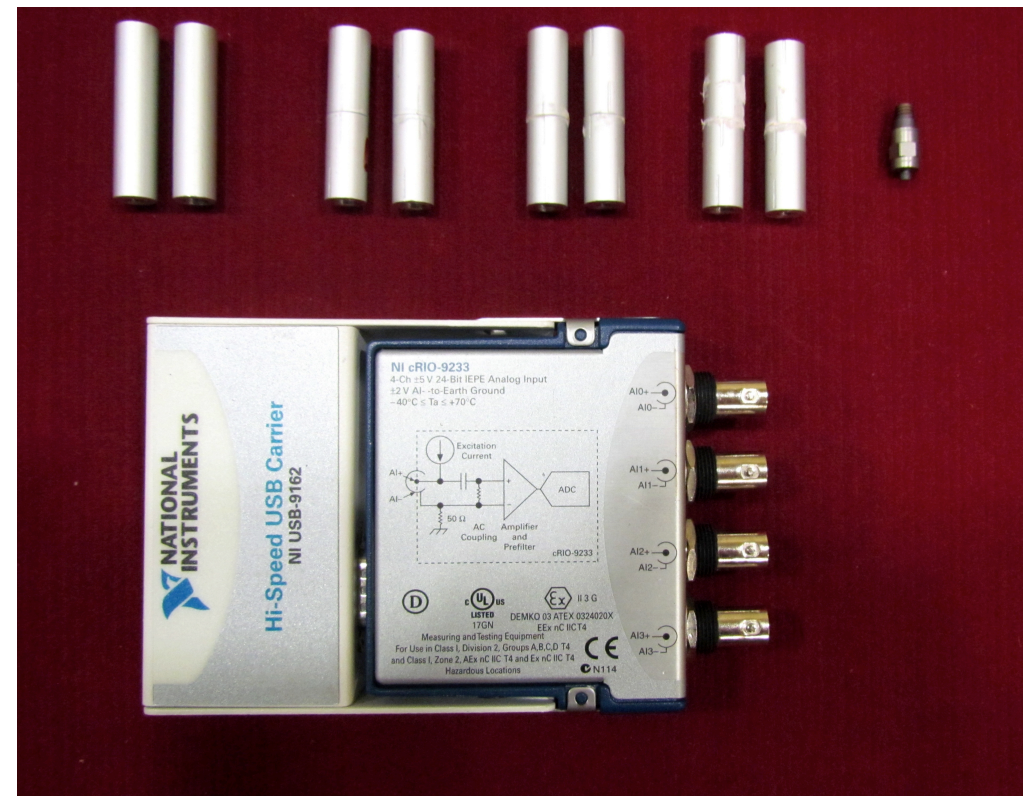

Figure 2. Experimental specimens, accelerometer and acquisition board

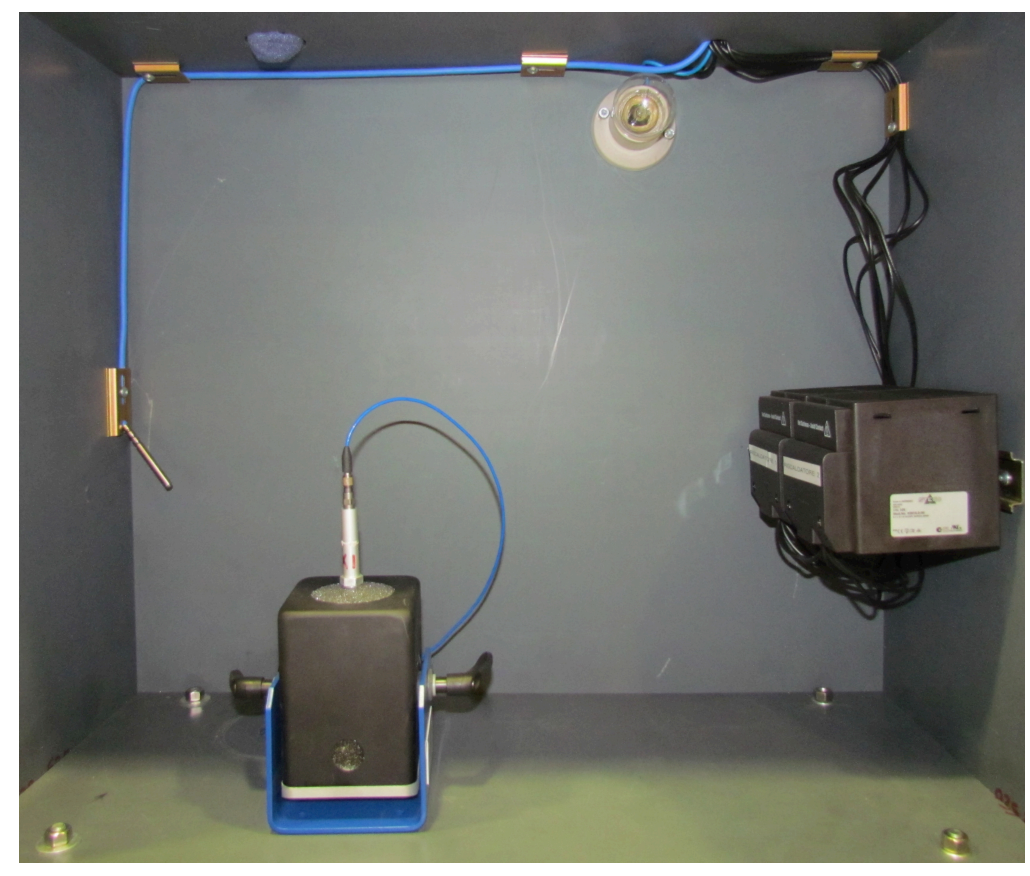

Figure 3. Experimental test rig with climatic chamber and mini shaker

perfect contact in each case. The model is implemented with linear hexahedral elements with standard formulation, 1223470 nodes and almost 3.5 million d.o.f. The need of having so much elements is driven by the very low thickness of the adhesive. In order to have undistorted elements in the adhesive layer, which is crucial for the analyses, we had to force a very refined mesh size in that region. This is a very common problem, as reported by many authors [26-28] The elastic moduli of the three adhesives are taken by the technical data sheets in literature and span from $100 \mathrm{MPa}$ for the Terostat 939 to $800 \mathrm{MPa}$ 
for the Loctite 454 and $4000 \mathrm{MPa}$ for the X60 - HBM adhesive. We considered room temperature for all the adhesives, leaving the evaluation of the temperature effect to the experimental part. The boundary condition applied is to prescribe at $0 \mathrm{~mm}$ the nodal displacement of the basis, as shown using orange triangles in Figure 4 in order to be consistent with the experimental tests. The frequency domain is limited to $20 \mathrm{kHz}$, in order to save computational time and do not exceed the experimental limit given by the mini-shaker. As output we can compare the natural frequencies of the bonded models with the frequency obtained using the reference and with the experimental results.

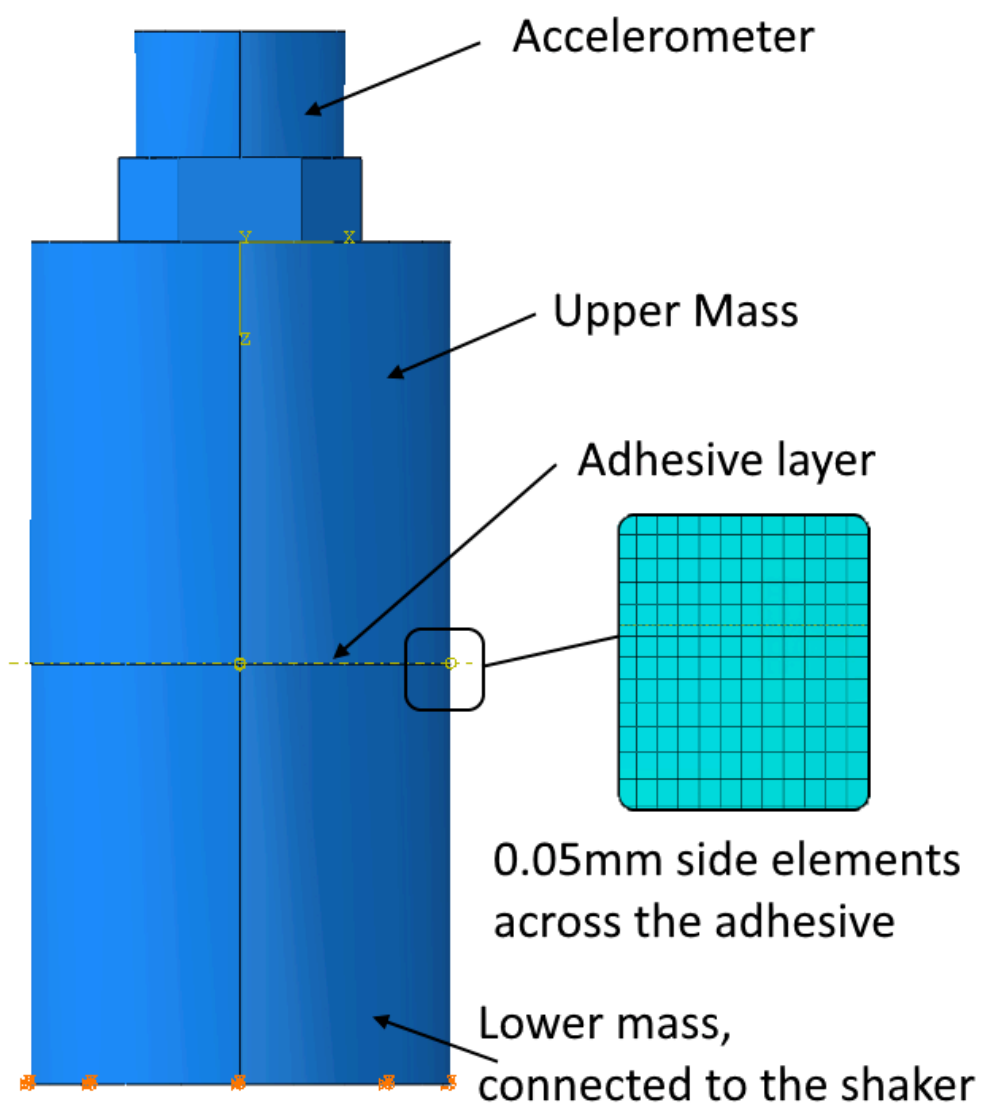

Figure 4. Boundary conditions applied to the FE model and magnification of the mesh of the adhesive layer

\section{RESULTS AND DISCUSSION}

\subsection{Finite element model results}

This section describes the finite element model results, showing the effect of the three materials and comparing the natural frequencies with the experimental results. The finite element model results can be qualitatively expressed by the modal form of the system and their natural frequencies. The modal forms of the systems are the same for all the four models and are reported, just for the Loctite 454 case, in Figure 5. It is important to note that with adopted linear perturbation analysis, the value of the magnitude 

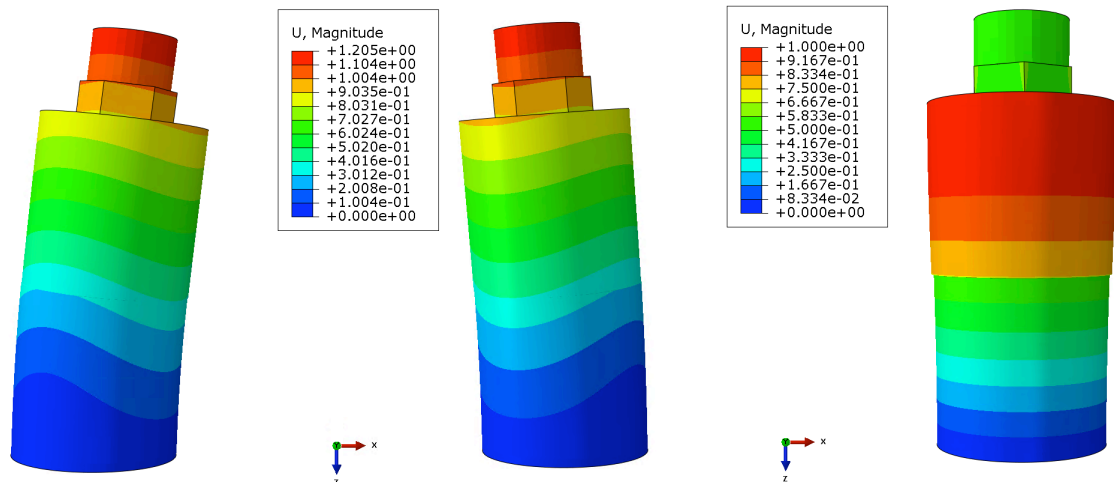

Figure 5. Modal forms of the system. From left to right, bending on first plane, bending on second plane, torsion along the axis

\begin{tabular}{|c|c|c|c|c|c|c|}
\hline Adhesive type & E (MPa) & ni & rho $(\mathbf{k g} / \mathbf{m 3})$ & 1st $(\mathbf{H z})$ & 2nd $(\mathbf{H z})$ & 3rd (Hz) \\
\hline Terostat 939 & 100 & 0.45 & 1500 & 6736.5 & 6736.6 & 17712 \\
\hline Loctite 454 & 800 & 0.3 & 1100 & 6738.3 & 6738.4 & 18262 \\
\hline HBM X60 & 4000 & 0.3 & 800 & 6889.7 & 6889.9 & 19046 \\
\hline None (reference) & - & - & - & 6926.8 & 6926.9 & 19234 \\
\hline
\end{tabular}

Table 3. Natural frequencies of the finite element model.

is not significant. The most important information is given by the frequencies of these three modal forms

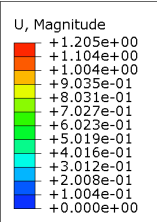

\subsection{Post-processing of the experimental data}

The post processing of the vibration data was done in National Instruments' LabVIEW 2016 environment. In order to avoid transient effect on the acquisition, the tails of the vibration signal are removed, i.e. 0.5 seconds at the beginning and at the end of the signal are removed from the 3 second of acquired data. The resulting $0.5 \mathrm{~Hz}$ of spectrum resolution allows to clearly match the excitation frequencies. The system response if computed by means of the power spectrum of the vibration data, as reported in equation 1 .

$$
P S(f)=\frac{F F T^{*}(\bar{x}) \cdot F F T(\bar{x})}{n^{2}}
$$


where $F F T(\bar{x})$ is the fast Fourier transform (FFT) of vibration data, while the $F F T^{*}$ denotes the complex conjugate and $n$ is the length of samples array. In an ideal condition, the sine input of the shaker - measured by the accelerometer - returns a single peak as output in the frequency domain, at the excitation frequency $f_{e x}$. An interesting comparison among different adhesive type is made on two scalar output parameters (despite connected):

- The amplitude of the power spectrum at the excitation frequency (SA).

- The ratio between the amplitude of the spectrum at the input frequency and the total energy of the acquired vibration signal (SE).

The choice of these parameters follows a simple reasoning: SA is what is usually measured in experimental activity, i.e., it is a measure of absolute value depending of the energy at input source, while SE returns the quality of the acquired signal independently of the energy of input source. A further consideration is given by The Parseval's theorem, regarding the choice of the power spectrum instead of a simple spectrum. In fact, the theorem states that the total energy contained in a time-domain waveform is equal to the total energy of the waveform' spectrum. The equality is reported in Equation 2.

$$
\int_{-\infty}^{+\infty}|x(t)|^{2} d t=\int_{-\infty}^{+\infty}|X(f)|^{2} d f
$$

It follows that the total energy of the signal can be computed as a simple sum of power spectrum components.

Two other outputs are computed dividing the values of the experimental points by the value of the reference configuration (on average), as mentioned in Section 2.2. The two parameters are named SAR and SER since they are derived from the SA and SE values respectively.

\subsection{Statistical analysis of the results: Analysis of Variance}

There are many statistical methodologies to tackle the analysis of multivariate problems [29]. Among these we adopted the Design of Experiment (DoE) procedure, a powerful statistical technique based on the analysis of variance (ANOVA) that can be conveniently applied to these classes of problems. ANOVA is based on the variance calculation (standard deviation) of a response considering the single variable and also the global variance of the responses. The ratio between these two quantities is called the F-value [29]. When we consider a random process this F-value equals one, meaning that the variable under analysis has no tangible effect on the selected response, since it behaves the same way as the experimental noise for practical test or numerical error for parametric simulation. On the contrary a larger F-value means can be associated to a variable that influences the process. Among the several approaches typically used to represent the results graphically one of the most popular is the normal plot. It is used mainly to demonstrate which are the main consequences of the variables on the system response, estimating whether a certain set of data follows a Gaussian distribution or not. When the data are approximately 
a straight line the response is statistically "normal" i.e. follows a stochastic law. When the selected variable strongly affects the system response its effect, reported in the normal plot, will then fall outside the normal distribution line. This line, also called errors line is built up thanks to the replicates of the experimental tests which have the variability typical of the experiment considered and their effect has a normal distribution. The replicates of the experiments are therefore important because they are used to build the error line because they are ruled by a stochastic law by definition. The greater the deviation of the point from the normal line the larger the confidence interval (i.e. the probability that the variables are significant is higher). In this paper we adopted the half normal plot, which behaves in the same way as the normal plot, but considers only the absolute significance of the variables and not their sign.

\subsubsection{Half-normal plot of the response}

Figure 6 shows the half-normal probability plots from an ANOVA following [17]. The analysis was performed on two outputs of the problem (system response) described in the previous subsection. Figure 6 shows the SA, Figure 7 the SE. In Figure 6 and 7 on the X-axis is represented the standardized effect linked to each factor considered. The higher the standardized effect, the greater the influence of the variable on the response. The Y-axis represents the half-normal probability associated with each effect. The greater the Y value the higher the probability that the effect has an influence on the problem. The solid line is the error line, which is obtained by interpolation of the replicates of the tests, represented by triangles, and also the high order interactions of the variables, represented by the squares, which do not play any role on the system response. The points that fall off the error line represent the factors that mainly affect each response. Figure 6 and 7 show that the frequency and the amplitude have the strongest influence both on SA and SE. The effect of the frequency is the most relevant both for SA and SE. It must be noted that we applied a transformation to the selected variables, in order to normalize the data. This linearization, called Box-Cox transformation, is strongly recommended [18] when there is the need of dealing with experimental set of data by means of ANOVA technique, which gives better prediction for linear problems. In Figure 6 the effect of the variables are reported applying an inverse square root transformation and in figure 7 we have SE raised to the power of 2.2. The amplitude is relevant in both cases, while the interaction between frequency and amplitude is more important for SE than SA. The half normal plots of the SAR and SER are reported in Figure 8 and Figure 9 respectively. These Figures show that dividing SA and SE by the reference configuration causes two different behaviors.

The SAR chart Figure 8 shows a strong dependence on the temperature (D) and on the interaction between frequency (A) and temperature (AD). The amplitude of the signal disappears when compared with Figure 6. The SER chart Figure 9 shows a more complex situation. In this case the amplitude and the frequency are important as it happens for the SE as shown in Figure 7 and in addition the temperature plays a secondary but still relevant role. 


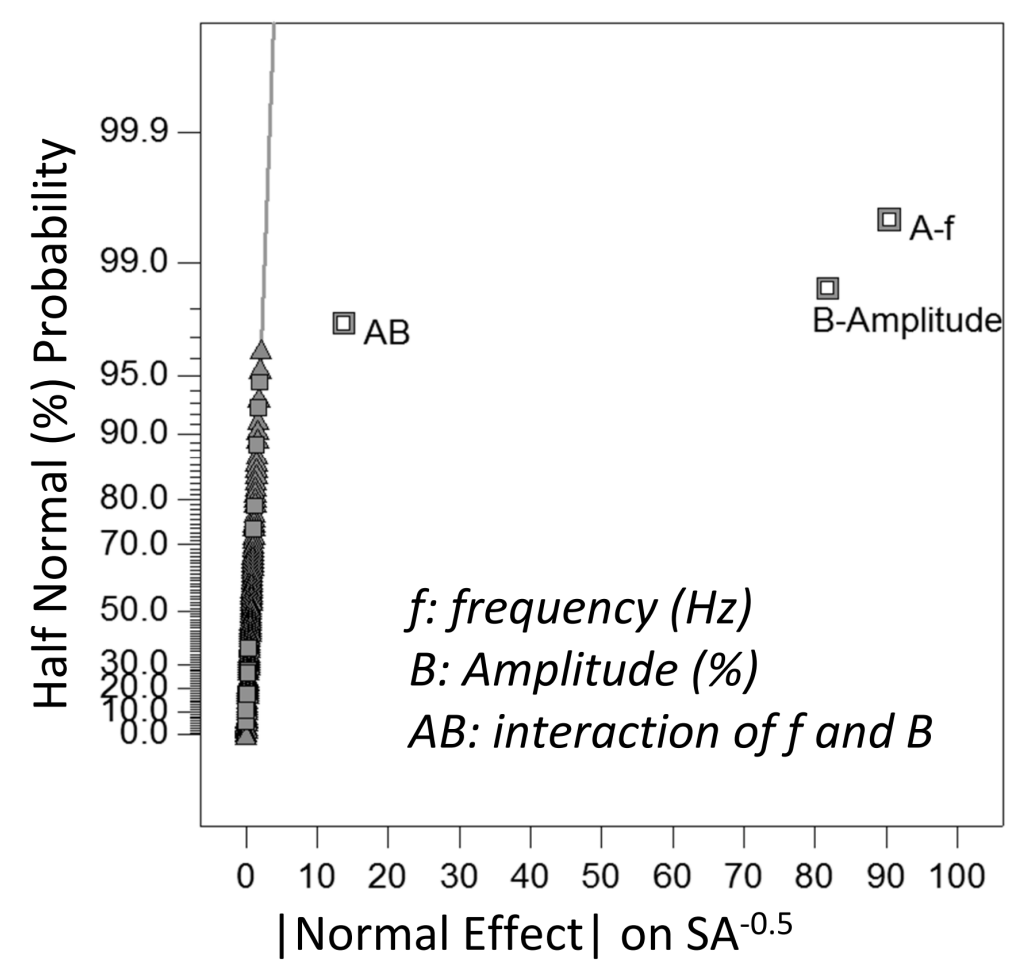

Figure 6. Half normal plot of the SA, with an inverse square root transformation applied

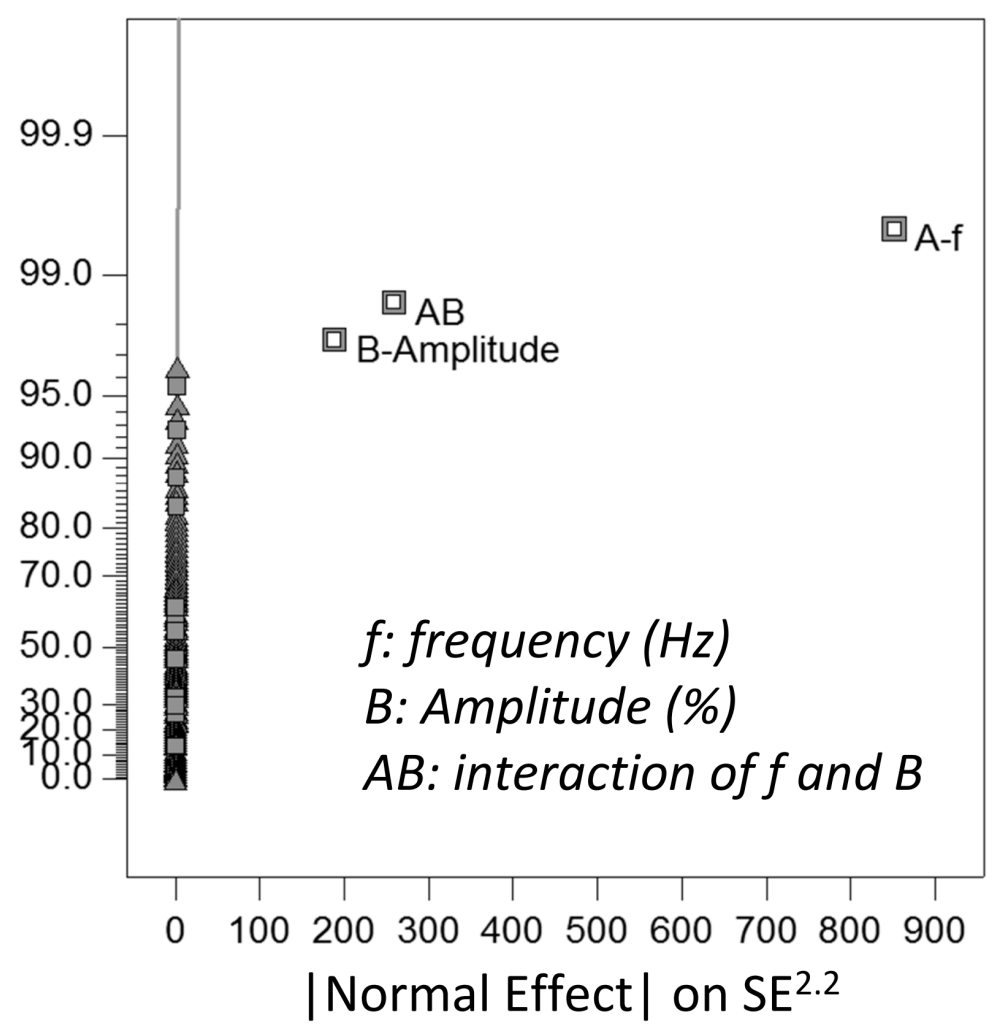

Figure 7. Half normal plot of the SE, with a power law transformation applied 


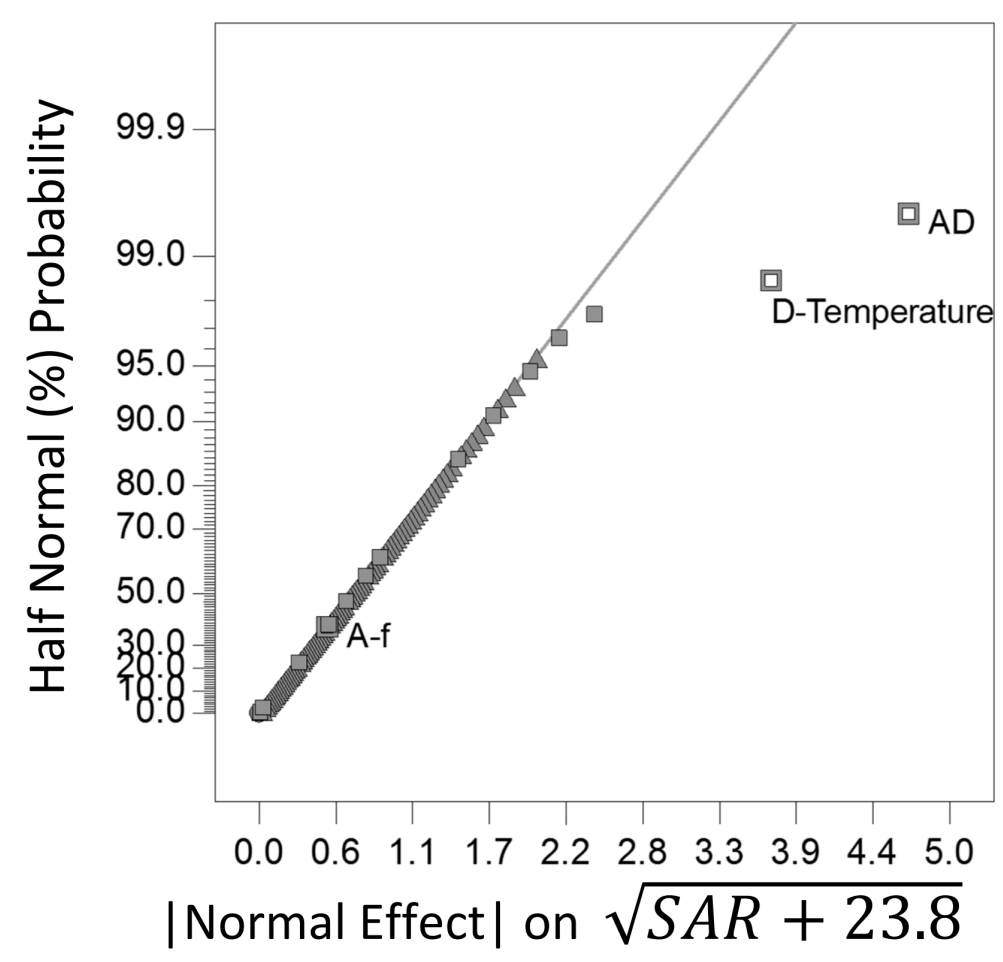

Figure 8. Half normal plot of the SAR

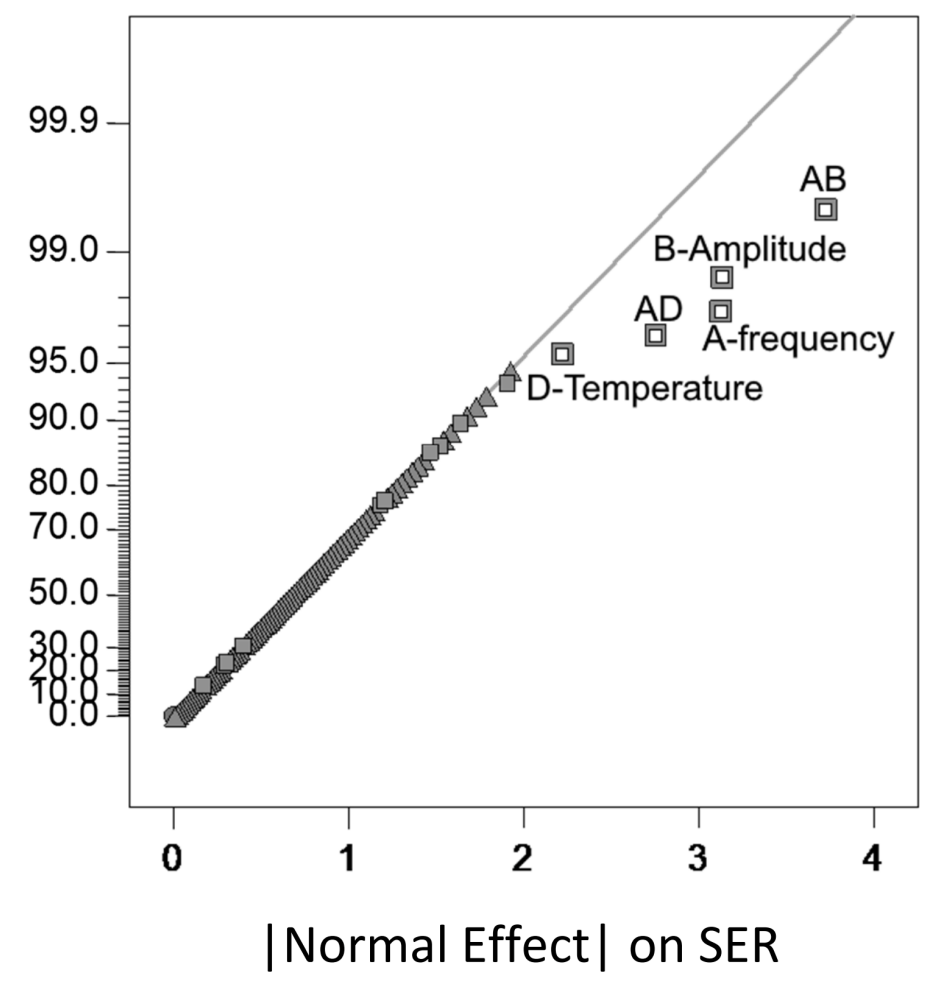

Figure 9. Half normal plot of the SER 


\subsubsection{Variable interactions and relevance}

Two main comments can be drawn by the graphs in Figure 6, 7,8 and 9. The first one is that the adhesive type is not relevant compared with the other variables. This is the most important finding of this research since it tells us that no matter the adhesive stiffness and the adhesive strength the bond between the excitation base and the accelerometer base is enough to transmit a correct signal. Therefore we can choose the most suitable adhesive according to other consideration such as cost, simplicity, availability and ease of application. The second important consideration is that the temperature somehow is relevant for SAR and SER. This means that it could be important to monitor the temperature as well as the vibration if we want to be sure of taking into account all the possible sources of variations. In order to show the direct influence on the four system response of the selected variables we can take advantage of the following graphs. Figure 10 shows that the system has larger value of SA with increasing frequency and amplitude. These two variables present an interaction clearly visible due to the divergence of the two interpolating lines. The points in the graph represent the experimental results obtained and it is visible the good repeatably and the scarce significance of the other variables. Figure 11 shows a completely different situation. The main effect on SE is at the lower frequency, where we can see a difference due to the amplitude. It must be noted that the mini-shaker at $15 \mathrm{~Hz}$ shows some issues, as reported in Figure 1. We did not expect a behavior like that, therefore we are investigating on this problem, to see if the behavior is due to the noise at low frequency. The SE parameter describes how many portion of the system's energy is transmitted at a given frequency with respect to the total one. Probably, the non linearities at low frequencies make the SE response sensible to the amplitude. It is worth noting that anyway no sign of a significant influence of the adhesive nor the temperature can be found as well. Disregarding the data at $15 \mathrm{~Hz}$ would lead to a substantial independence of SE on the other variables. Figure 12 shows the effect of the temperature and of the frequency on the SAR. In this case, in order to show the actual values we did not apply the Box-Cox transformation as we did in Figure 8. The figure 12 shows three curves, as a function of the test temperature. The red squares represent the $15 \mathrm{~Hz}$ tests, the green triangles depict the $3500 \mathrm{~Hz}$ tests and the blue dots the $7000 \mathrm{~Hz}$ tests. The adhesive and the amplitude have no effect, but they appear only as the error bars in the the three curves. As for the SE the SER results, shown in Figure 13 is affected by the results at $15 \mathrm{~Hz}$. We can easily spot a difference in the the two amplitude and frequencies as a function of the temperature, but it is much more evident at $15 \mathrm{~Hz}$ rather than at the other frequencies. It is useful to highlight that, even though there is some noise at $15 \mathrm{~Hz}$, the experimental tests show a clear absence of the adhesive in each statistical analysis performed. This is the main outcome the authors wanted to find out and therefore the conclusion is that the bonding do not change the vibratory signal from the source to the sensor, even considering soft adhesive such as the Terostat 939 or when the test temperature increases up to $80^{\circ} \mathrm{C}$. 


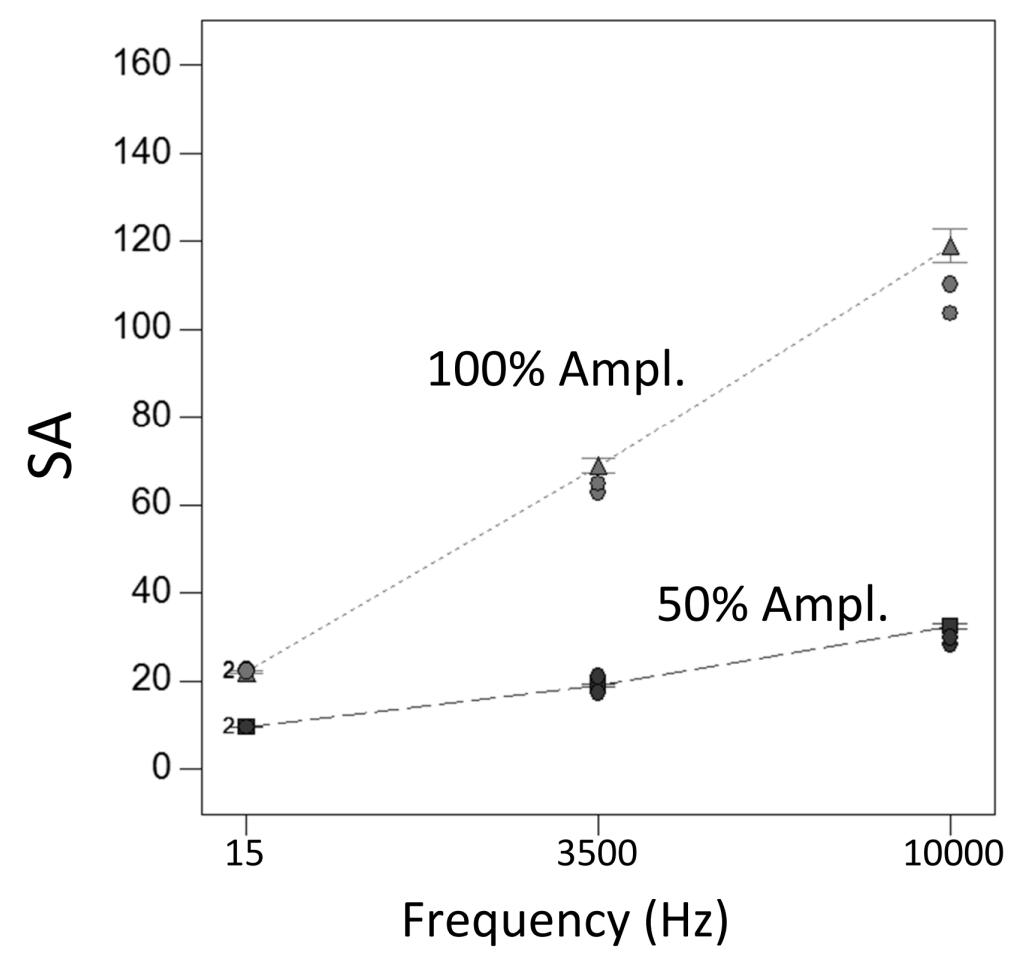

Figure 10. Effect of the main variables on SA

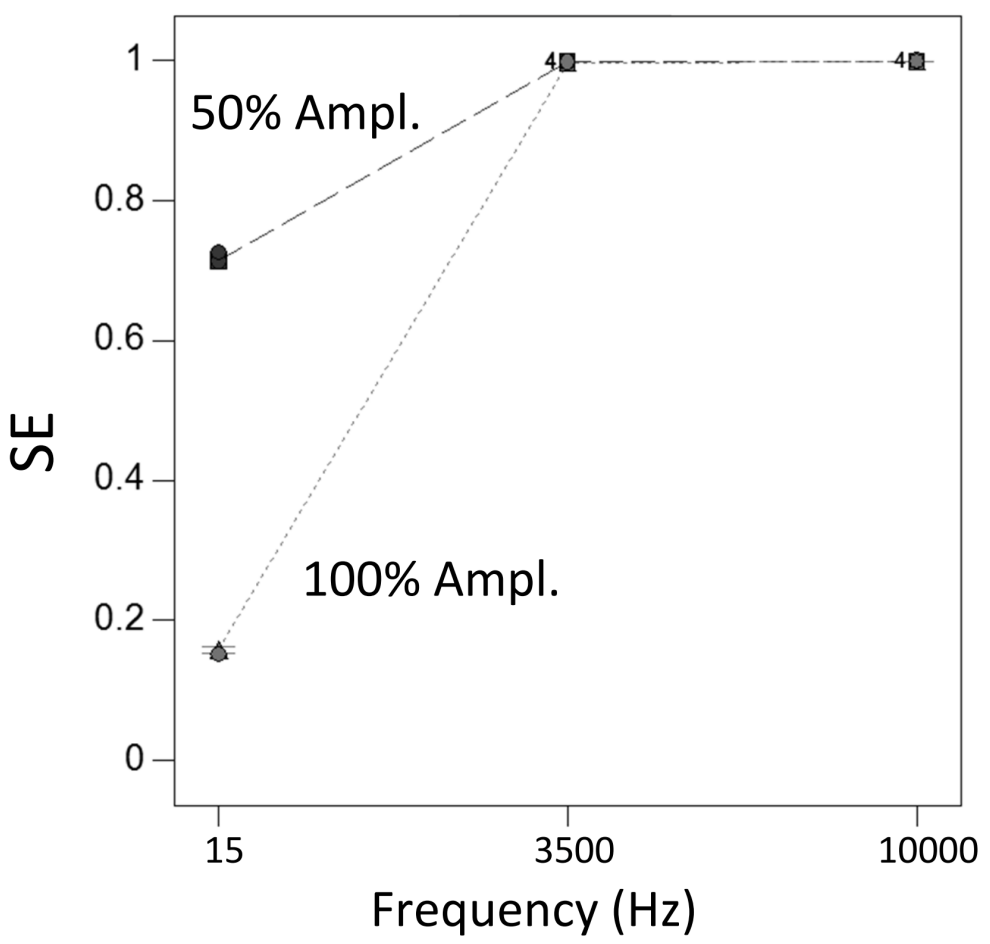

Figure 11. Effect of the main variables on SE 


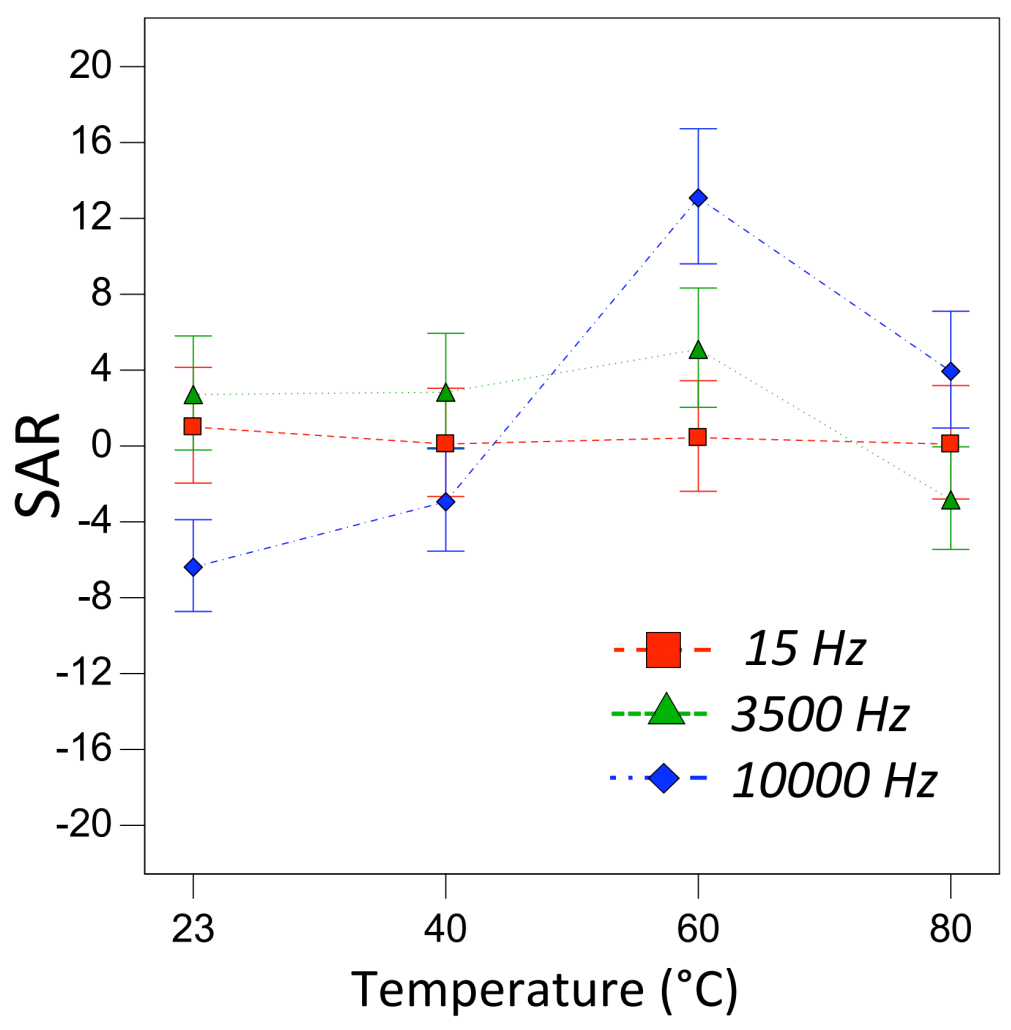

Figure 12. Effect of the main variables on SAR

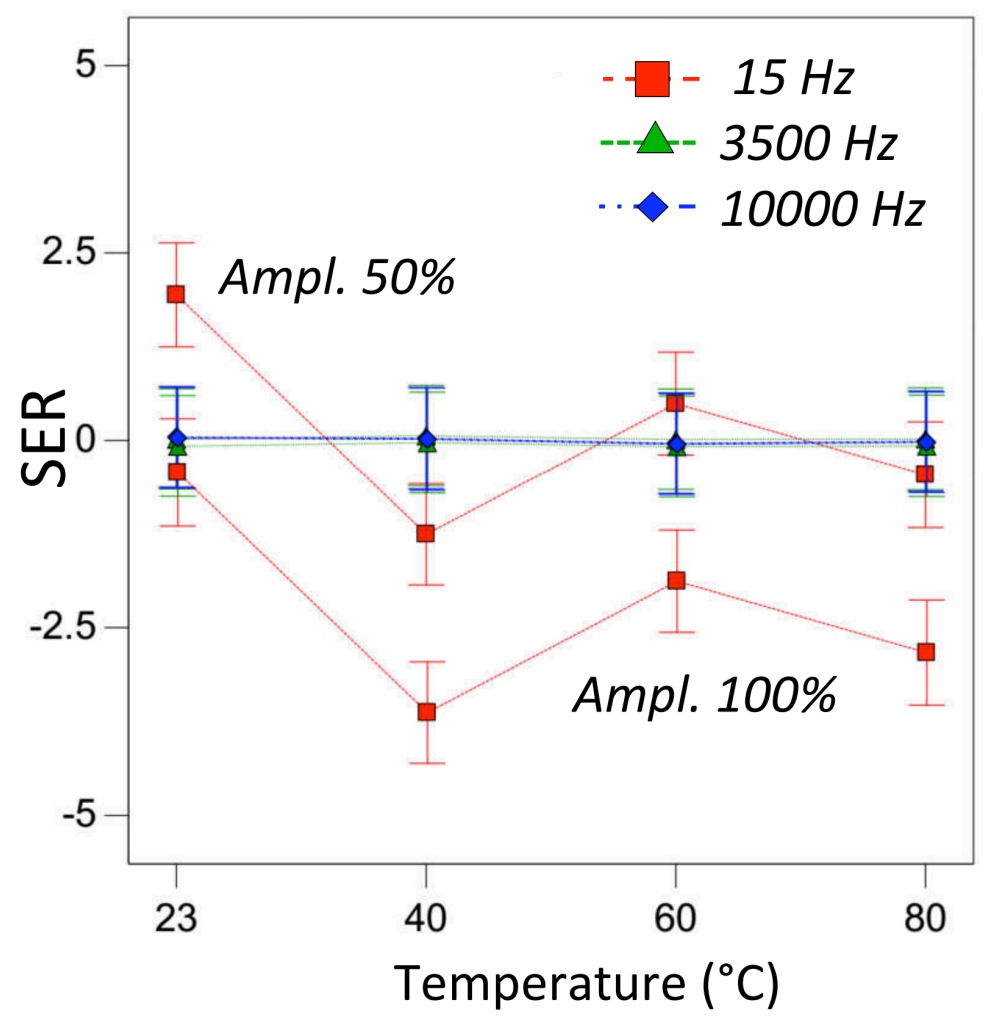

Figure 13. Effect of the main variables on SER 


\section{CONCLUSION}

This paper focuses on the effect of temperature on the frequency response function (FRF) of three different structural adhesives, namely a stiff two component methylmethacrylate (HBM X60), a soft modified silane (Terostat 939) and a cyanoacrylate superglue (Loctite 454). The methylmethacrylate is commonly used in strain gauge application and accelerometers setup in on-field environments due to its stiffness. The cyanoacrylate is the most used in accelerometers setup for diagnostics purposes in laboratory due to its fast polymerization time. The silane is a general purpose adhesive chosen for its different structural characteristics compared to the other two. They have been used to joint two aluminium cylinders, one connected to an accelerometer and the other to the head of an electromagnetic shaker. A reference additional experiment has been carried on a double-weight aluminium base, simply obtained using a continuum block of double length without any adhesive. The experimental setup is placed inside a climatic chamber providing a selectable temperature up to $80^{\circ} \mathrm{C}$. The shaker provides a sinusoidal vibration in terms of acceleration at different combinations of frequency and amplitude, as summarised in Table 1. A monoaxial accelerometer is used to acquire the feedback vibration signal which has been processed to compute four different parameters assessing the dynamic response of the structure. The output parameters are all computed through the power spectrum of the vibratory signal.They are: SA, i.e the amplitude of the spectrum at the shaker excitation frequency, SE, i.e the ratio between SA and the total energy of the signal, SAR and SER i.e. the values of SA and SE for a given adhesive compared to the corresponding values in the reference condition. The numerical model developed shows that the presence or absence of the adhesive layer does not modify or disturb the natural frequency of the system.

A design of experiments (DOE) approach was used to extract the main conclusions which are summarized as follows:

- Frequency and amplitude have the strongest influence on both SA and SE

- The temperature has a strong influence on SAR

- The SER is influenced by the frequency, the amplitude and the temperature

- The amplitude and temperature have an evident effect on the SA

- No sign of a significant influence of the adhesive nor the temperature can be found on SE, SAR and SER

Above them two results are can be used as design guidelines: no matter the adhesive stiffness and the adhesive strength the bond between the excitation base and the accelerometer base is enough to transmit a correct signal. Therefore the most suitable adhesive can be chosen according to other consideration such as cost, simplicity, availability and ease of application. The temperature somehow is relevant for SAR and SER. This means that it could be important to monitor the temperature as well as the vibration if we want to be sure of taking into account all the possible sources of variations. 


\section{COMPLIANCE WITH ETHICAL STANDARD}

Conflict of Interest: The authors declare that they have no conflict of interest.

\section{REFERENCES}

[1] C. Hellier, Handbook of Nondestructive Evaluation, Second Edition. 2012.

[2] C. Aszkler, "Acceleration, Shock and Vibration Sensors," in Sensor Technology Handbook, pp. 137159,2005

[3] S. Bowers, K. Piety, and R. Piety, "Real World Mounting of Accelerometers for Machinery Monitoring," Sound and Vibration, vol. 25, no. 2, pp. 14-23, 1991.

[4] Dytran, "Dytran Accelerometer Mounting Considerations," 2015.

[5] PCB, "Introduction to Piezoelectric Accelerometers," 2015.

[6] MMF, "Metra Mess und Frequenztechnik in Radebeul,” 2015.

[7] C. Harris, A. Piersol, and T. Paez, Harris'shock and vibration handbook. 2002.

[8] M. Cocconcelli and A. Spaggiari, "Mounting of accelerometers with structural adhesives: experimental characterization of the dynamic response," The Journal of Adhesion, vol. 8464, no. March, pp. 1-14, 2015.

[9] Hbm X60 Datasheet, "Instructions for use Superglue X 60," 2013.

[10] Henkel, "Loctite 454 Technical Datasheet," 2012.

[11] Henkel, “Terostat 737 Technical Datasheet,” 2015.

[12] R. D. Adams and N. A. Peppiatt, "Stress analysis of adhesive-bonded lap joints," The Journal of Strain Analysis for Engineering Design, vol. 9, no. 3, pp. 185-196, 1974.

${ }^{[13]}$ E. Koricho, E. Verna, G. Belingardi, B. Martorana, and V. Brunella, "Parametric study of hot-melt adhesive under accelerated ageing for automotive applications," International Journal of Adhesion and Adhesives, vol. 68, pp. 169-181, 2016.

[14] Z. Jia, D. Hui, G. Yuan, J. Lair, K.-t. Lau, and F. Xu, "Mechanical properties of an epoxy-based adhesive under high strain rate loadings at low temperature environment," Composites Part B: Engineering, vol. 105, pp. 132-137, 2016.

[15] A. K. Kadiyala and J. Bijwe, "Investigations on performance and failure mechanisms of high temperature thermoplastic polymers as adhesives," International Journal of Adhesion and Adhesives, vol. 70, pp. 90-101, 2016.

[16] L. F. da Silva and R. Adams, "Adhesive joints at high and low temperatures using similar and dissimilar adherends and dual adhesives," International Journal of Adhesion and Adhesives, vol. 27, pp. 216-226, 42007. 
[17] D. C. Montgomery, "Design and Analysis of Experiments," Design and Analysis of Experiments, p. John Wiley and Sons, 2004.

[18] R. Mead, The Design of Experiments: Statistical Principles for Practical Applications. Cambridge University Press, 1990.

[19] PCB, “Technical Datasheet PCB Accelerometer 353B18_N,” 2017.

[20] D. Castagnetti, A. Spaggiari, and E. Dragoni, "Robust Shape Optimization of Tubular Butt Joints for Characterizing Thin Adhesive Layers under Uniform Normal and Shear Stresses," Journal of Adhesion Science and Technology, vol. 24, pp. 1959-1976, 12010.

[21] J. J. Cognard, R. Créac'hcadec, L. Sohier, P. Davies, R. Creac\&\#39;Hcadec, L. Sohier, R. Creachcadec, P. Davies, L. Sohier, and P. Davies, "Analysis of the nonlinear behavior of adhesives in bonded assemblies-Comparison of TAST and Arcan tests," International Journal of Adhesion and Adhesives, vol. 28, pp. 393-404, 122008.

[22] L. Sohier and P. Davies, "Analysis of the nonlinear behavior of adhesives in bonded assemblies Comparison of TAST and Arcan tests," International Journal of Adhesion and Adhesives, vol. 28, pp. 393-404, 2008.

[23] A. Spaggiari, E. Dragoni, and H. F. Brinson, "Measuring the shear strength of structural adhesives with bonded beams under antisymmetric bending," International Journal of Adhesion and Adhesives, vol. 67, pp. 112-120, 2016.

[24] K. S. Kwan, The Role of Penetrant Structure on the Transport and Mechanical Properties of a Thermoset Adhesive. PhD thesis, Blacksbrurg, VirginaTech, 81998.

[25] M. J. Anderson and P. J. Whitcomb, DOE Simplified: Practical Tools for Effective Experimentation, Third Edition. Productivity Press, 3rd ed., 2015.

[26] D. Castagnetti, A. Spaggiari, and E. Dragoni, "Efficient finite element modeling of the static collapse of complex bonded structures," International Conference on CRACK PATHS (CP 2009), 2009.

[27] D. Castagnetti, E. Dragoni, and A. Spaggiari, "Failure analysis of bonded T-peel joints: Efficient modelling by standard finite elements with experimental validation," International Journal of Adhesion and Adhesives, vol. 30, no. 5, pp. 306-312, 2010.

[28] M. Tsai and J. Morton, "An evaluation of analytical and numerical solutions to the single-lap joint," International Journal of Solids and Structures, vol. 31, pp. 2537-2563, 91994.

[29] P. Ito, "7 Robustness of ANOVA and MANOVA test procedures," Handbook of Statistics, vol. 1, pp. 199-236, 1980. 